\title{
A Preliminary Study on the Feasibility of the Quantitative Parameters of Dual-Energy Computed Tomography Enterography in the Assessment of the Activity of Intestinal Crohn's Disease
}

\author{
Wei-xiong Xiao* \\ Yu-ting Zhu* \\ Zhi-chao Zhang \\ Min Luo \\ Ming-ping $\mathrm{Ma}$
}

Department of Radiology, Fujian Provincial Hospital, Provincial Clinical College of Fujian Medical University, Fuzhou, People's Republic of China

*These authors contributed equally to this work
Correspondence: Min Luo; Ming-ping Ma Department of Radiology, Fujian Provincial Hospital, Provincial Clinical College of Fujian Medical University, No. 134 East Street, Gulou District, Fuzhou, 35000I, People's Republic of China

Tel +8659187557768

Email radiodrluo@।63.com
Objective: To investigate the value of dual energy CT enterography (DECTE) in evaluating the activity of Crohn's disease (CD).

Methods: The endoscopy and imaging data of 29 patients with CD confirmed by clinic and pathology were analyzed retrospectively. The clinical CD activity index (CDAI) was used as the disease activity grouping standard, 29 patients with $\mathrm{CD}$ were grouped into activity groups, 18 patients in the active group $(\mathrm{CDAI} \geq 150)$ with 36 intestinal segments, and 11 patients in the remission group (CDAI < 150) with 20 intestinal segments. The virtual single energy CT value, slope of energy spectrum curve and iodine content were analyzed to evaluate the evaluation of intestinal CD activity by DECTE.

Results: There were statistically significant differences in virtual single energy CT value (except $90 \mathrm{keV}$ and $100 \mathrm{keV}$ virtual single energy CT value), curve slope and iodine content between remission group and active group $(\mathrm{P}<0.05)$, and has more diagnostic value for active phase (AUC > 0.5). (1) Virtual single energy CT value: the AUC of $60 \mathrm{keV}$ in arterial phase was the highest (0.924). The specificity of diagnosing CD in active stage was high (95\%). (2) Curve slope: the AUC of portal vein phase was the largest (0.731). The specificity of diagnosing CD in active stage was higher (85\%). (3) Iodine content: the AUC of arterial phase was the highest $(0.885)$. The specificity of diagnosing CD lesions in the active stage was $100 \%$.

Conclusion: The virtual single energy CT value, energy spectrum curve slope and iodine content can provide reference for clinical accurate diagnosis of CD activity.

Keywords: Crohn's disease, computed tomography, dual energy CT, virtual single

\section{Introduction}

Crohn's disease (CD) is a chronic granulomatous disease of unknown origin that alternates between exacerbation and remission, with gastrointestinal disease as a clinical manifestation. According to the guidelines, ${ }^{1}$ The diagnosis of $\mathrm{CD}$ is based on a combination of clinical presentation and endoscopic, radiologic, histologic, and pathologic findings that demonstrate some degree of focal, asymmetric, and transmural granulomatous inflammation of the luminal GI tract and the clinical treatment options during active and remission stages of CD should differ. The active phase can be treated with amino salicylic acid agents, immunosuppressive agents, biological agents alone or in combination with systemically acting hormones to 
induce remission depending on the degree of CD activity, and patients with complex severe $\mathrm{CD}$ can be treated with surgical interventions.

The small bowel is one of the most common areas affected by inflammation in patients with CD. Much of the inflammation is beyond the reach of standard endoscopic evaluation. Therefore, the correct determination of the active and remission stages through imaging information can help in the selection of appropriate treatment, especially for those patients with contraindication to endoscopic evaluation. CTE has a reported sensitivity as high as $90 \%$ in detecting lesions associated with CD. While MRE has similar sensitivity to CTE with wall enhancement, mucosal lesions, and T2 hypersensitivity as suggestive of intestinal inflammation. ${ }^{1,2}$

Dual-energy computed tomography enterography (DECTE) uses the difference in virtual monoenergetic CT values of different substances to localize and capture an image of an irradiated object in two-dimensional energy space. The most commonly used base substance pairs are iodine and water. The decomposition algorithm can be used to calculate the relative contribution of iodine and water components within a substance, thus enabling the identification and quantitative analysis of the properties of the substance.

Although the activity of $\mathrm{CD}$ varies with the degree of congestion, edema, and fibrosis of the intestinal wall, material separation and quantitative analysis can be achieved through DECTE. The present study aimed to investigate the value and feasibility of the quantitative parameters of DECTE in the assessment of the activity of intestinal $\mathrm{CD}$ in both its remission and active stages.

\section{Materials and Methods}

\section{Subjects}

The data of 29 patients (Table 1) diagnosed with CD between July 1, 2016, and December 31, 2019, in the Department of Gastroenterology, Fujian Provincial Hospital, were retrospectively collected and subjected to double-phase DECTE enhancement scans. In each patient, the diseased intestinal segments (mucosal hyperenhancement, wall thickening (small intestine $\geq 3 \mathrm{~mm}$, large intestine $\geq 5 \mathrm{~mm}$ ), mural stratification with a prominent vasa recta (comb sign), mesenteric fat stranding, and Extra-intestinal complications (fistulas, peri-intestinal abscesses $)^{1-5}$ )was selected for the observation group (when there was more than one diseased segment, up to four segments were selected at the same time), and the normal intestinal segments (with an intestinal lumen diameter of $<2.5 \mathrm{~cm}$ and intestinal wall thickness of $<3 \mathrm{~mm}$ without morphological signs of $\mathrm{CD}$ ) were selected for the control group, resulting in a total of 56 segments being included.

Inclusion criteria: (1) Patients who met the diagnostic criteria for $\mathrm{CD}$ as recommended by the American Gastrointestinal Association's clinical guidelines (2018); ${ }^{1}$ (2) patients without contraindications for enhanced CT scanning.

Exclusion criteria: (1) Patients with a history of surgical resection; (2) patients who underwent a capsule endoscopy to examine their small intestine but failed to discharge the capsule, or patients with any obvious local image artifacts that might affect observation; (3) patients with poor bowel preparation that might affect observation and measurement.

The present study complied with the ethical principles of medical research set out in the Declaration of Helsinki and was approved by the ethics committee of Fujian Provincial Hospital.

\section{Examination Methods}

Preparation before the examination: (1) One day before the examination, the patient was fed a semi-liquid diet with little residue and received intestinal preparation. (2) 1.5 hours before the examination, $1500 \mathrm{~mL}$ of $2 \%$ isotonic mannitol solution was administered orally to the patient (in the case of no contraindication) to fill the stomach and duodenum. (3) 15 minutes before the examination, the patient (in the case of no contraindication) was given an intramuscular injection of $20 \mathrm{mg}$ of anisodamine (654-2) to inhibit intestinal motility. (4) $800 \mathrm{~mL}$ of $2 \%$ mannitol solution was infused through the patient's anal canal to fill the colon (in the case of no contraindication). Bowel preparation was not conducted in patients with intestinal

Table I Summary of Clinical Information Related to a Total of 56 Intestinal Segments in 29 Crohn's Patients

\begin{tabular}{|c|c|c|c|c|c|c|c|c|}
\hline & \multicolumn{2}{|c|}{ Gender } & \multirow[t]{2}{*}{ Age } & \multicolumn{3}{|c|}{ Lesioned Intestinal Segment } & \multicolumn{2}{|c|}{ Inflammatory Indicators } \\
\hline & $\mathbf{M}$ & $\mathbf{F}$ & & Small Intestine & Ileocecum & Colon & CRP (mg/L) & ESR $(\mathrm{mm} / \mathrm{L})$ \\
\hline Active group & 12 & 6 & $32.8 \pm 14.6$ & 20 & 11 & 5 & $6 I \pm 35.97$ & $49.61 \pm 25.49$ \\
\hline Remission group & 10 & 1 & $31.1 \pm 9.6$ & 9 & 8 & 3 & $9.23 \pm 10.41$ & $15.9 \pm 8.73$ \\
\hline
\end{tabular}


obstruction, and the examination was conducted under gastrointestinal decompression. For patients with a weak constitution, the enema was conducted without the oral administration of isotonic mannitol solution.

A Somatom Force dual-source CT (Siemens, Germany) was used for the scans. The patient was instructed to hold their breath, and volume scanning was conducted from the top of the diaphragm to the symphysis pubis.

Parameters of the DECTE scanning: The A/B tube voltage was $\mathrm{Sn} 80 \mathrm{kVp} / \mathrm{Sn} 150 \mathrm{kVp}$, respectively, and the A/B tube current was $130 \mathrm{mAs} / 65 \mathrm{mAs}$, respectively. The speed was $0.5 \mathrm{~s} / \mathrm{r}$, and the pitch was $0.6 .1 .2 \mathrm{~mL} / \mathrm{kg}$ of the contrast agent ioversol $(320 \mathrm{mg} / \mathrm{mL}) .15 \mathrm{~mL}$ of $0.9 \% \mathrm{NaCl}$ solution was used for the enhancement scanning. After scanning, the reconstructed thin-layer data with a thickness of $1.5 \mathrm{~mm}$ were transferred to a postprocessing workstation (syngo.via VB10, Siemens, Germany) for dual-energy image data analysis.

\section{Image Analysis and Processing}

The results of the clinical disease activity index (CDAI) were completed independently by a physician with associate seniority or higher in the Department of Gastroenterology. The DECTE results were completed by two residents and the associate chief of the Department of Radiology. The target bowel segment was determined by two residents independently; when there was disagreement on the selected target segment, the final decision was made by the associate chief physician through a review of the films. Both examinations used a double-blind method.

The scanned arterial phase and portal phase Sn80 kVp and $\mathrm{Sn} 150 \mathrm{kVp}$ images were transferred to a Siemens postprocessing workstation (syngo.via VB10) with the adoption of the dual-energy module. The monoenergetic module was selected for energy-spectrum analysis, and the most obvious lesion on the mesenteric side of the observation group in transverse, coronal, or sagittal position was selected. A circular region of interest (ROI) with a range of $70-80 \%$ of the overall intestinal wall was outlined, with avoidance of the tissues outside the intestinal wall. The energy spectrum curve was automatically drawn by the system, and the CT values of $40-100 \mathrm{keV}$ were measured with adjustment of $\mathrm{keV}$. The liver NVC module was used to obtain an iodine chart, and the appropriate ROI was selected. The same processing was performed on the segments in the control group at the same time. Measurements were taken three times by two residents independently and consecutively in three layers, and the mean of both was recorded.

\section{Statistical Analysis}

SPSS 25.0 was used for data processing. A ShapiroWilk test was used to test the normality of all measured data, and those conforming to a normal distribution were described as mean \pm standard deviation; those that did not were described as median \pm interquartile range. An independent sample $t$-test or a Mann-Whitney $U$-test was used to compare the different virtual monoenergetic CT values, the slope of energy-spectrum curve $\mathrm{K}$, and iodine content. $P=0.05$ was considered the standard value. The receiver operating curve (ROC) was plotted and the area under the curve (AUC Area Under Curve) calculated.

\section{Results}

\section{Clinical Data}

Data concerning age, gender, disease duration, CDAI, C-reactive protein (CRP), erythrocyte sedimentation rate (ESR), and type of diseased intestinal segment were collected from the enrolled patients. Of the 29 cases that were enrolled, 22 were male and 7 were female. The ages of the enrolled patients ranged between 15 and 80 years, with an average age of $32.8 \pm 12.8$ years. The disease duration ranged between 0.5 and 10 years, with an average duration of $3.7 \pm 3.0$ years. Of the 56 diseased intestinal segments included, 29 were from the small intestine, 19 were ileocecal, and 8 were from the colon. A total of 36 bowel segments were placed in the active group (CDAI $\geq 150$ ), with an average CRP of 61 $\pm 35.97 \mathrm{mg} / \mathrm{L}$ and an average ESR of $49.61 \pm 25.49 \mathrm{~mm} / \mathrm{L}$, and a total of 20 segments were placed in the remission group (CDAI < 150), with an average CRP of $9.23 \pm 10.41 \mathrm{mg} / \mathrm{L}$ and an average ESR of $15.9 \pm 8.73 \mathrm{~mm} / \mathrm{L}$.

\section{Image Data}

Comparison of Different Virtual Monoenergetic CT Values, The Slope of Energy-Spectrum Curve K, and lodine Content Between the Control Group (The Normal Intestinal Segments) and the Observation Group (The Diseased Intestine Segments) (Table 2) Analysis of the characteristics of the DECTE intestinal wall energy spectrum (Figures $1 \mathrm{~F}, \mathrm{~J}, 2 \mathrm{~F}$ and $\mathrm{J}$ ) revealed that the $\mathrm{CT}$ value of the intestinal wall in the virtual monoenergetic map in the observation group decreased with the increase of $\mathrm{keV}$; the decrease was faster between 40 and $100 \mathrm{keV}$, with a larger slope, and the slope between 
Table 2 Summary of Different Virtual Single Energy CT Values, Slope K of Energy Spectrum Curve and lodine Content in Diseased Intestine and Normal Intestine

\begin{tabular}{|c|c|c|c|c|c|c|c|c|c|c|}
\hline & & $40 \mathrm{Kev}$ & $50 \mathrm{Kev}$ & $60 \mathrm{Kev}$ & $70 \mathrm{Kev}$ & $80 \mathrm{Kev}$ & $90 \mathrm{Kev}$ & $100 \mathrm{Kev}$ & $\mathbf{K}$ & $\begin{array}{l}\text { lodine } \\
\text { Content }\end{array}$ \\
\hline \multirow[t]{4}{*}{$\begin{array}{l}\text { Arterial } \\
\text { phase }\end{array}$} & $\begin{array}{c}\text { Observation } \\
\text { group }\end{array}$ & $207.9 \pm 44.3$ & $147.5 \pm 29.7$ & $111.9 \pm 20.1$ & $90.1 \pm 14.6$ & $76.2 \pm 11.6$ & $65.9 \pm 9.1$ & $60.2 \pm 8.7^{*}$ & $2.5 \pm 0.7$ & $2.3 \pm 1.0$ \\
\hline & Control group & $109.1 \pm 22.9 *$ & $76.8 \pm 16.8$ & $62.4 \pm 12.6$ & $53.4 \pm 10.6 *$ & $48.3 \pm 8.5^{*}$ & $43.9 \pm 7.1^{*}$ & $40.6 \pm 7.9 *$ & $1.1 \pm 0.3$ & $1.0 \pm 0.3$ \\
\hline & $\mathrm{T} / \mathrm{Z}$ & -8.775 & 15.495 & 15.594 & -8.804 & $-8.74 I$ & -8.636 & -8.287 & 13.743 & 9.569 \\
\hline & $P$ & 0 & 0 & 0 & 0 & 0 & 0 & 0 & 0 & 0 \\
\hline \multirow[t]{4}{*}{$\begin{array}{l}\text { Portal vein } \\
\text { phase }\end{array}$} & $\begin{array}{c}\text { Observation } \\
\text { group }\end{array}$ & $275.4 \pm 52.6$ & $191.1 \pm 34.5$ & $144.6 \pm 23.8$ & $112.0 \pm 16.5$ & $92.2 \pm 13.6$ & $78.1 \pm 10.3$ & $69.3 \pm 9.2$ & $3.4 \pm 0.9$ & $2.8 \pm 0.6$ \\
\hline & Control group & $142.2 \pm 30.9 *$ & $103.2 \pm 15.3^{*}$ & $80.0 \pm 13.1 *$ & $65.8 \pm 10.4^{*}$ & $55.5 \pm 9.2$ & $48.9 \pm 8.0$ & $44.1 \pm 8.0$ & $1.6 \pm 0.4$ & $1.7 \pm 0.5^{*}$ \\
\hline & $T / Z$ & -8.793 & -8.79 & -9.026 & -9.008 & $|6.74|$ & $|6.78|$ & 15.35 & 14.4 & -8.51 \\
\hline & $P$ & 0 & 0 & 0 & 0 & 0 & 0 & 0 & 0 & 0 \\
\hline
\end{tabular}

Note: *Indicates that the data does not fit a normal distribution.

100 and $190 \mathrm{keV}$ was parallel to the horizontal axis. The curves of the two phases of the intestinal wall in the control group were close in position and were all located below the curves of the intestinal wall in the observation group. The comparison suggested that there were statistically significant differences between the observation group and the control group in the virtual monoenergetic CT values, the slope of energy-spectrum curve $\mathrm{K}$, and iodine content.
Comparison of Different Virtual Monoenergetic CT Values, The Slope of Energy-Spectrum Curve K, and lodine Content Between the Remission Group and the Active Group (Table 3)

No statistically significant difference was found between the remission group and the active group in the $\mathrm{CT}$ values of the intestinal wall at the portal phase of 90 and $100 \mathrm{keV}(P>0.05$ in both). There were, however, statistically significant differences in the CT values, slope of energy-spectrum curve $\mathrm{K}$,

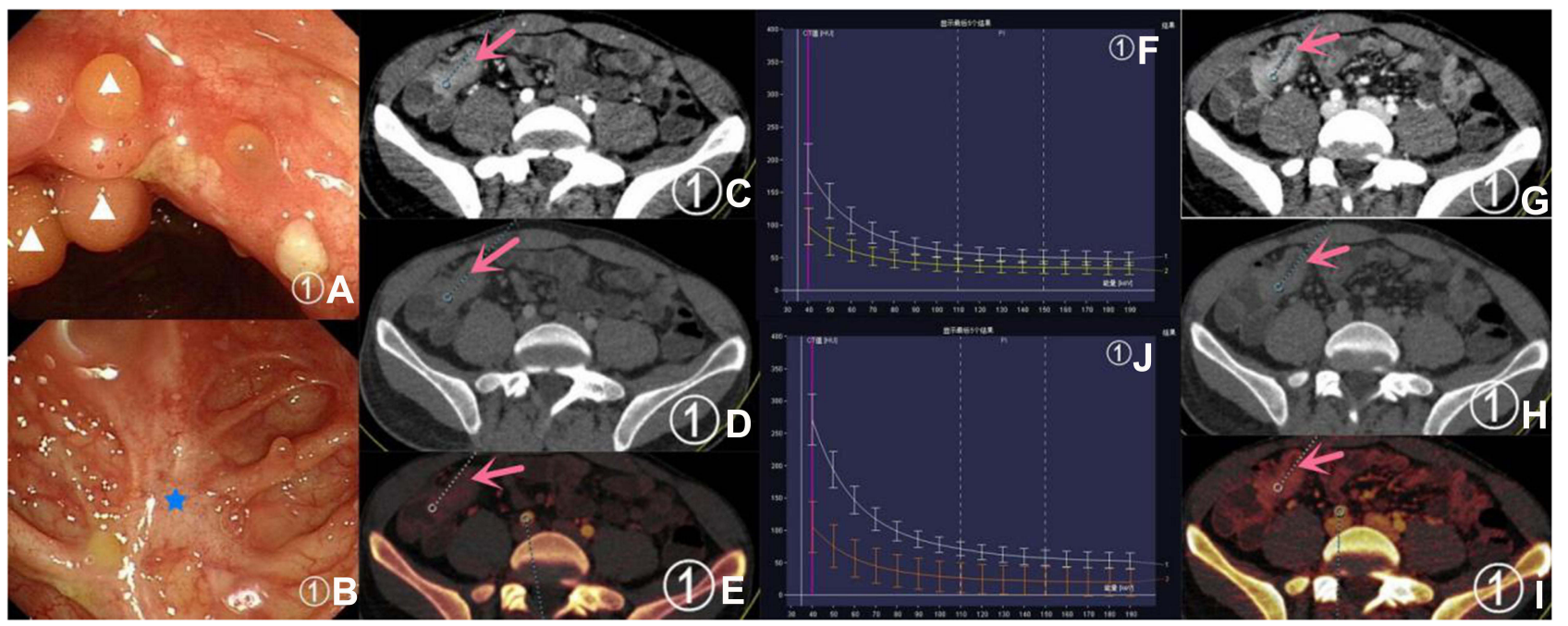

Figure I A 27-year-old male patient with the main complaint of a change in the nature of his stools and abdominal pain for more than two years. CDAI = 80.I (remission stage). ( $\mathbf{A}$ and $\mathbf{B}$ ) show the endoscopic manifestations: the pentagonal markers indicate white scars and triangular markers indicate pseudogranulomas. (C and $\mathbf{D})$ show the virtual monoenergetic charts, in the arterial phase, of $40 \mathrm{keV}$ and $100 \mathrm{keV}$, respectively; the CT values of the diseased intestinal segment (pink arrow) were I86.8 HU and $67.7 \mathrm{HU}$, respectively. ( $\mathbf{G}$ and $\mathbf{H}$ ) show the virtual monoenergetic charts, in the portal phase, of $40 \mathrm{keV}$ and $\mathrm{I} 00 \mathrm{eV}$, respectively; the CT values of the diseased intestinal segment (pink arrow) were 27I.3 HU and $78 \mathrm{HU}$, respectively. (E and I) show the iodograms in the arterial and portal phase, respectively; the iodine contents of the diseased intestinal segment (pink arrow) were $2.1 \mathrm{mg} / \mathrm{mL}$ and $2.9 \mathrm{mg} / \mathrm{mL}$, respectively. (F and J) show the arterial and portal energy-spectrum curves, respectively, with the diseased bowel segment at the top and the normal bowel segment at the bottom. 
Table 3 Comparison of Different Virtual Single Energy CT Values, K and lodine Contents in the Intestinal Wall Between the Remission Group and the Active Group

\begin{tabular}{|c|c|c|c|c|c|c|c|c|c|c|}
\hline & & $40 \mathrm{Kev}$ & $50 \mathrm{Kev}$ & $60 \mathrm{Kev}$ & $70 \mathrm{Kev}$ & $80 \mathrm{Kev}$ & $90 \mathrm{Kev}$ & $100 \mathrm{Kev}$ & $\mathbf{K}$ & $\begin{array}{l}\text { lodine } \\
\text { Content }\end{array}$ \\
\hline \multirow[t]{4}{*}{$\begin{array}{l}\text { Arterial } \\
\text { phase }\end{array}$} & $\begin{array}{c}\text { Remission } \\
\text { group }\end{array}$ & $168.1 \pm 31.9$ & $119.6 \pm 20.8$ & $93.0 \pm 14.8$ & $76.9 \pm 11.6$ & $66.2 \pm 9.8$ & $58.9 \pm 9.1$ & $53.7 \pm 8.4$ & $2.2 \pm 0.7$ & $1.5 \pm 0.5$ \\
\hline & $\begin{array}{l}\text { Active } \\
\text { group }\end{array}$ & $230.0 \pm 33.6 *$ & $|63.0 \pm 2| .4$ & $122.4 \pm 14.2$ & $97.4 \pm 10.5$ & $81.8 \pm 8.3$ & $69.8 \pm 6.5$ & $62.8 \pm 6.3^{*}$ & $2.7 \pm 0.7$ & $2.7 \pm 0.9$ \\
\hline & $T / Z$ & -6.713 & -7.345 & -7.336 & -6.745 & -6.351 & -5.238 & -3.942 & -2.329 & -5.633 \\
\hline & $P$ & 0 & 0 & 0 & 0 & 0 & 0 & 0 & 0.024 & 0 \\
\hline \multirow{4}{*}{$\begin{array}{l}\text { Portal } \\
\text { vein } \\
\text { phase }\end{array}$} & $\begin{array}{c}\text { Remission } \\
\text { group }\end{array}$ & $235.3 \pm 42.1$ & $170.3 \pm 27.8$ & $130.3 \pm 23.2$ & $98.3 \pm 27.9 *$ & $86.4 \pm 14.5$ & $74.8 \pm 11.3$ & $67.6 \pm 11.1$ & $3.1 \pm 0.6$ & $2.4 \pm 0.4$ \\
\hline & $\begin{array}{l}\text { Active } \\
\text { group }\end{array}$ & $297.7 \pm 44.3$ & $202.7 \pm 32.6$ & $152.5 \pm 20.3$ & $117.0 \pm 20.3$ & $95.4 \pm 12.2$ & $79.9 \pm 9.4$ & $70.2 \pm 8.1$ & $3.7 \pm 0.9$ & $3.0 \pm 0.5$ \\
\hline & $T / Z$ & -5.136 & -3.742 & -3.72 & -2.821 & -2.479 & -1.816 & -0.99 & -2.668 & -5.226 \\
\hline & $P$ & 0 & 0 & 0 & 0 & 0.016 & 0.075 & 0.327 & 0.01 & 0 \\
\hline
\end{tabular}

Note: *Indicates that the data does not fit a normal distribution.

and iodine content of the intestinal wall at other $\operatorname{keVs}(P<$ 0.05 in all), and the CT values, slope of energy-spectrum curve $\mathrm{K}$, and iodine content of the intestinal wall were higher in the active group than in the remission group.

A 27-year-old male patient with the main complaint of a change in the nature of his stools and abdominal pain for more than two years. CDAI $=80.1$ (remission stage), with endoscopy, pathology, arterial phase 40 and $100 \mathrm{Kev}$ virtual monoenergetic images and iodine map (Figure 1A-E, G-I).Meanwhile,A 32-year-old male patient with the main complaint of recurrent abdominal pain for more than a year. CDAI $=380$ (active stage), with endoscopy, pathology, arterial phase 40 and $100 \mathrm{Kev}$ virtual monoenergetic images and iodine map (Figure 2A-E, $\mathrm{G}-\mathrm{I}$ ). In the active group, the enhancement of the "diseased bowel wall" at $40 \mathrm{Kev}$ in the arterial phase was

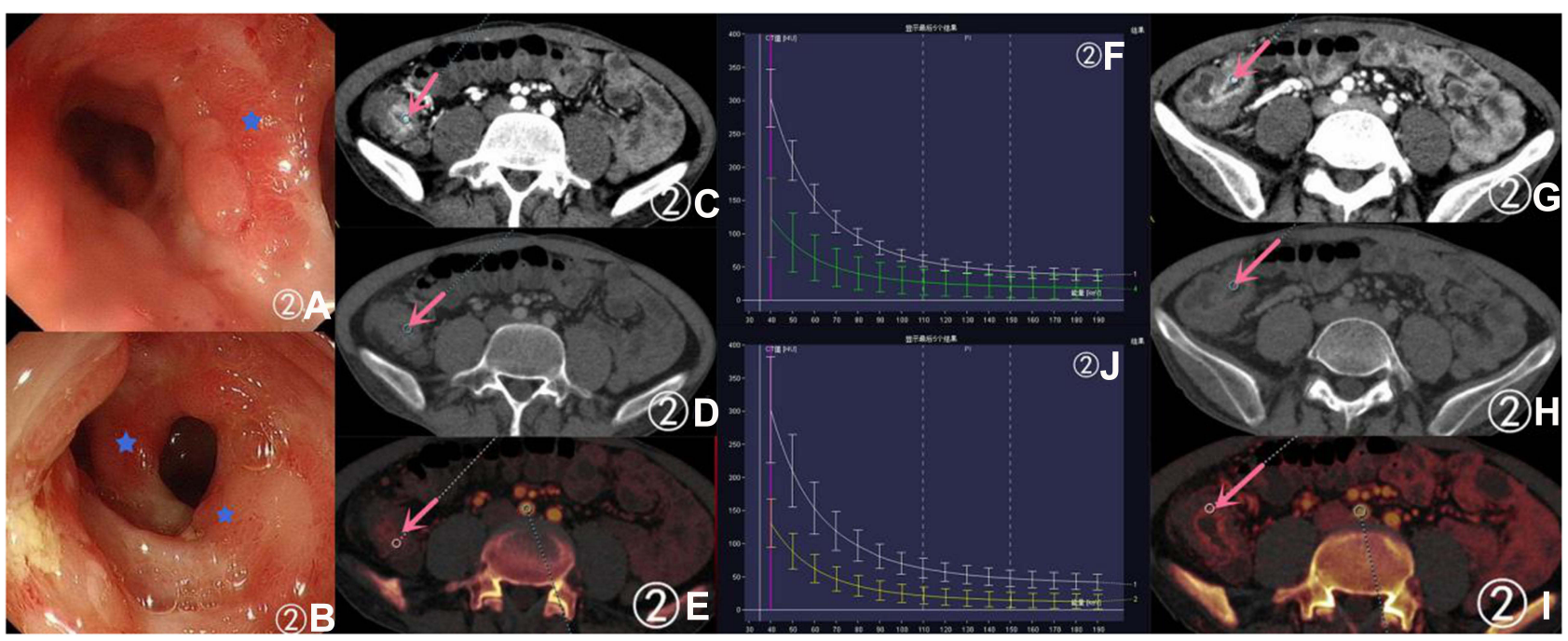

Figure 2 A 32-year-old male patient with the main complaint of recurrent abdominal pain for more than a year. CDAI = 380 (active stage). (A and B) show the endoscopic manifestations: the pentagonal markers indicate congestion and edema. (C and $\mathbf{D}$ ) show the virtual monoenergetic charts, in the arterial phase, of $40 \mathrm{keV}$ and $100 \mathrm{keV}$, respectively; the CT values of the diseased intestinal segment (pink arrow) were $303.5 \mathrm{HU}$ and $67 . \mathrm{I} \mathrm{HU}$, respectively. (G and $\mathbf{H})$ show the virtual monoenergetic charts, in the portal phase, of $40 \mathrm{keV}$ and $100 \mathrm{keV}$, respectively; the CT values of the diseased intestinal segment (pink arrow) were $3 \mathrm{I} 4.5 \mathrm{HU}$ and $73 . \mathrm{I} \mathrm{HU}$, respectively. (E and I) show the iodograms in the arterial and portal phase, respectively; the iodine contents of the diseased intestinal segment (pink arrow) were $3.3 \mathrm{mg} / \mathrm{mL}$ and $3.6 \mathrm{mg} / \mathrm{mL}$, respectively. ( $\mathbf{F}$ and $\mathbf{J}$ ) show the arterial and portal energy-spectrum curves, respectively, with the diseased bowel segment at the top and the normal bowel segment at the bottom. 
Table 4 The Value of Different Virtual Single Energy CT Values, K and lodine Content in the Assessment of CD Activity in Arterial and Portal Phases

\begin{tabular}{|c|c|c|c|c|c|c|c|c|}
\hline & \multicolumn{4}{|c|}{ Arterial Phase } & \multicolumn{4}{|c|}{ Portal Vein Phase } \\
\hline & AUC & $\begin{array}{l}\text { Threshold Value } \\
\text { (HU) }\end{array}$ & $\begin{array}{c}\text { Sensitivity } \\
\text { (\%) }\end{array}$ & $\begin{array}{c}\text { Specificity } \\
\text { (\%) }\end{array}$ & AUC & $\begin{array}{l}\text { Threshold Value } \\
\text { (HU) }\end{array}$ & $\begin{array}{c}\text { Sensitivity } \\
\text { (\%) }\end{array}$ & $\begin{array}{c}\text { Specificity } \\
(\%)\end{array}$ \\
\hline $40 \mathrm{kev}$ & 0.894 & 211.8 & 69.4 & 95 & 0.853 & 244.3 & 88.9 & 70 \\
\hline $50 \mathrm{kev}$ & 0.919 & 132.7 & 94.4 & 75 & 0.799 & 166.4 & 91.7 & 65 \\
\hline $60 \mathrm{kev}$ & 0.924 & III.I & 75 & 95 & 0.783 & 131.9 & 83.3 & 70 \\
\hline $70 \mathrm{kev}$ & 0.906 & 88.6 & 80.6 & 90 & 0.729 & 105.3 & 77.8 & 70 \\
\hline $80 \mathrm{kev}$ & 0.899 & 74.4 & 83.3 & 85 & 0.693 & 88.8 & 75 & 70 \\
\hline $90 \mathrm{kev}$ & 0.846 & 65.2 & 72.2 & 90 & 0.643 & 74.3 & 77.8 & 60 \\
\hline $100 \mathrm{kev}$ & 0.82 & 58.5 & 80.6 & 85 & 0.6 & 62.7 & 83.3 & 50 \\
\hline $\mathrm{K}$ & 0.690 & 2.62 & 66.7 & 75 & 0.731 & 3.6 & 58.3 & 85 \\
\hline lodine content & 0.885 & 2.55 & 61.1 & 100 & 0.843 & 2.75 & 77.8 & 90 \\
\hline
\end{tabular}

more pronounced than in the remission group, and iodine content of the intestinal wall were higher in the active group than in the remission group.

\section{The Values of Different Virtual Monoenergetic CT} Values, The Slope of Energy-Spectrum Curve K, and lodine Content in the Assessment of the Activity of CD (Table 4)

The ROC curves (Figure 3) of the three quantitative parameters in the active group and remission group were plotted separately. The results suggested that virtual monoenergetic CT values, energy-spectrum curve $\mathrm{K}$, and iodine content were of higher diagnostic value in the active stage of $\mathrm{CD}$ than in the remission stage (AUC $>0.5$ in all).

Different virtual monoenergetic CT values: At $60 \mathrm{keV}$ in the arterial phase, the different virtual monoenergetic $\mathrm{CT}$ values were highest at $\mathrm{AUC}=0.924$; at this time point, when the virtual monoenergetic CT value was $\geq 111.1 \mathrm{HU}$, the sensitivity and specificity of the virtual monoenergetic CT values for diagnosing the bowel segment in patients with $\mathrm{CD}$ in the active stage were $75 \%$ and $95 \%$, respectively.

Energy-spectrum curve K: In the portal phase, the slope of the energy-spectrum curve $\mathrm{K}$ was highest at $\mathrm{AUC}=0.731$; at this time point, when $\mathrm{K}$ was $\geq 3.6$, the sensitivity and specificity of the energy-spectrum curve $\mathrm{K}$ for diagnosing the bowel segment in patients with $\mathrm{CD}$ in the active stage were $58.3 \%$ and $85 \%$, respectively.

Iodine content: In the arterial phase, the iodine content was highest at $\mathrm{AUC}=0.885$; at this time point, when iodine content was $\geq 2.55$, the sensitivity and specificity of the iodine content for diagnosing the bowel segment in patients with $\mathrm{CD}$ in the active stage were $61.1 \%$ and $100 \%$, respectively. (See Figures 1-3.)
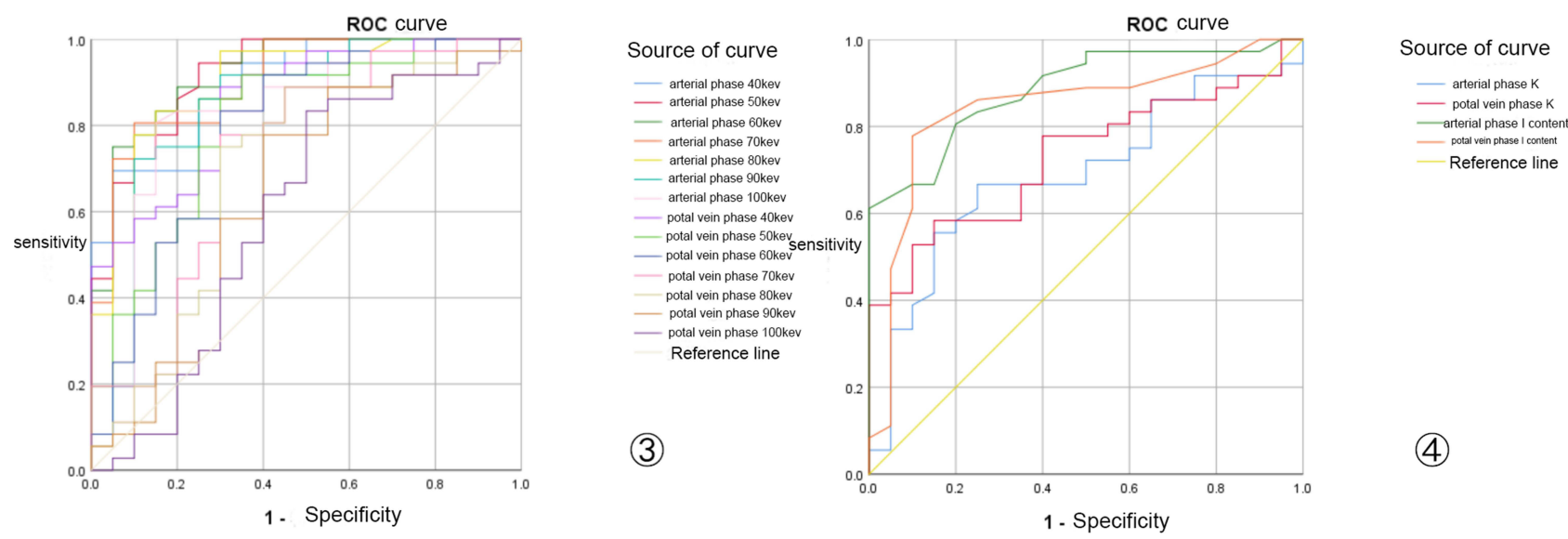

Figure 3 (3) and (4) show the ROC curves of the virtual monoenergetic CT values, slope of the energy-spectrum curve, and iodine content in the active stage and in the remission stage, respectively. 


\section{Discussion}

$\mathrm{CD}$ is a chronic granulomatous disease of unknown etiology, and treatment options vary with different degrees of activity. Therefore, how the activity of CD is assessed is critical. Although there are numerous methods for the assessment of CD activity, there is not yet a single gold standard. CDAI is still the main scoring system for the activity of $\mathrm{CD}$, but although it is used as a criterion for assessing the efficacy of drug therapy in $\mathrm{CD}$, it has limitations. The $\mathrm{CD}$ endoscopic index of severity (CDEIS) is more refined, but it is not suitable for patients with combined intestinal strictures; it is also painful, complex, time-consuming, inconvenient, and does not allow observation of the entire intestinal wall and peristomal conditions. Ultrasound examination is convenient and fast but has higher requirements for the physician. Magnetic resonance enterography does not use radiation, but the contents of the intestinal cavity affect image quality, so greater intestinal preparation and a longer examination duration are required, leading to higher costs. CTE is also commonly used in the diagnosis of CD, but, although it has a short examination duration, the activity of $\mathrm{CD}$ can only be assessed using morphology.

In recent years, many studies have looked at the separation function of DECTE substances, mostly focusing on analysis and differentiation using quantitative parameters, such as iodine value and energy-spectrum profile. $^{2-12}$ DECTE scanning technology offers evaluation with the adoption of morphological results and improved diagnostic accuracy with the virtual monoenergetic spectrum CT values, energy-spectrum curve slope, and iodine content of organ and tissues as well as other quantitative parameters obtained by post-processing. The increased contrast in low $\mathrm{keV}$ images may help with lesion identification, and the increased signal-to-noise ratio in high $\mathrm{keV}$ images may assist with detailed observation. Taguchi et $\mathrm{al}^{9}$ concluded that the $\mathrm{CT}$ values of the intestinal wall in the virtual monoenergetic chart decreased with increasing $\mathrm{keV}$ due to the photoelectric effect, that the sensitivity and specificity of intestinal wall $\mathrm{CT}$ values were higher at 40 $\mathrm{keV}$, and that the portal phase was better than the arterial phase. Another study ${ }^{12}$ found that virtual scanning can replace conventional plain scanning. Therefore, DECTE scans can eliminate the need for conventional CT plain scans and two-phase enhancement, resulting in a significant reduction in scan duration and radiation dose. Moreover, the use of iodograms (iodine content graph) can quantify the blood supply to diseased tissues and more accurately reflect the changes in the blood supply of the tissue.

De Kock et $\mathrm{al}^{4}$ suggested that there was a difference in the iodine content of the intestinal wall between the active and remission stages of $\mathrm{CD}$, and Liu et $\mathrm{a}^{11}$ suggested that iodine values in dual-energy $\mathrm{CT}$ parameters might reflect $\mathrm{CD}$ activity to some extent. The present study indicates that the reason for this might be that the formation of inflammatory congestion, inflammatory cell infiltration, and non-caseous granuloma in the diseased intestinal wall could lead to changes in the tissue type of the intestinal wall. The degree of mucosal injury, intestinal wall congestion, edema, and fibrosis in the diseased intestinal segment during the remission stage and the active stage of CD might also be different, which would make the intestinal wall's absorption and attenuation to X-rays different between the observation group and the control group and between the remission group and the active group, resulting in different characteristics of the energy spectrum. The difference in blood supply to the intestinal wall between the observation group and the control group and between the remission group and the active group might also lead to differences in the iodine content of the examined tissues. In this study, in addition to investigating the differences in energy spectrum curves and slopes between "diseased bowel segments" and "normal bowel segments", we also further investigated the differences in energy spectrum curves and slopes between the active and remission groups and their values for assessing $\mathrm{CD}$ activity.

The present study found that the virtual monoenergetic CT values, slope of the energy-spectrum curve, and iodine content were higher in the active stage of $\mathrm{CD}$ than in the remission stage, probably because the combing sign was obvious in the active stage of $\mathrm{CD}$, with the intestinal wall being inflamed and congested and an increased blood supply and iodine content in the tissues, resulting in higher CT values. In the remission stage, the intestinal wall was mainly infiltrated by chronic inflammatory cells and fibrosis, and the degree of inflammatory congestion in the active stage was not as high, so the blood supply might not be as obvious as in the active stage and the iodine content in the tissues not as high. This was also evidenced by the presence of the combing sign during the active stage and the degree of elevated inflammatory indicators.

The present study also found that the diagnostic values of the virtual monoenergetic CT values, slope of the energyspectrum curve, and iodine content were higher in the active stage of CD than in the remission stage. This may be due to the fact that, in the active stage of $\mathrm{CD}$, the intestinal wall 
undergoes an acute inflammatory reaction, and the degree of increased vascular permeability, congestion, and edema of the intestinal wall is more obvious than in the remission stage; the degree of fibrosis, however, is more obvious in the remission stage than in the active stage. Peng et $\mathrm{al}^{6}$ suggested that the mucosal injury, intestinal wall congestion, edema, and fibrotic changes of intestinal segments in different active stages of CD differed and that there were differences in the quantitative parameters of dual-energy CT and the characteristics of energy-spectrum curves, which is consistent with the results of the present study. Compared to MRE, DECTE has a lower price and shorter examination time, making it easier for patients to cooperate. The DECTE scan technique provides an accurate assessment of the activity of CD patients by combining morphological signs and multiple quantitative parameters, especially for those patients with contraindication to endoscopic evaluation.

The present study had some limitations. The adoption of CDAI as a criterion for classifying the activity of CD was highly subjective, and the number of enrolled cases was limited, so further studies in prospective trials with large samples are needed.

\section{Conclusion}

CD is an inflammatory lesion of the whole intestinal wall, and its activity is closely correlated with its treatment and prognosis. The present study showed that virtual monoenergetic CT values, energy-spectrum slope, and iodine content have good diagnostic value, sensitivity, and specificity in the assessment of the activity of CD, thereby providing a reference for accurate clinical diagnosis.

\section{Ethics Approval and Consent to Participate}

This study was conducted in accordance with the declaration of Helsinki.This study was conducted with approval from the Ethics Committee of Fujian Provincial Hospital. A written informed consent was obtained from all participants.

International Journal of General Medicine

\section{Publish your work in this journal}

The International Journal of General Medicine is an international, peer-reviewed open-access journal that focuses on general and internal medicine, pathogenesis, epidemiology, diagnosis, monitoring and treatment protocols. The journal is characterized by the rapid reporting of reviews, original research and clinical studies

\section{Disclosure}

The authors report no conflicts of interest in this work.

\section{References}

1. Lichtenstein GR, Loftus EV Jr, Isaacs KL, Regueiro MD, Gerson LB, Sands BE. Correction: ACG clinical guideline: management of Crohn's disease in adults. Am J Gastroenterol. 2018;113(7):1101. doi:10.1038/s41395-018-0120-x

2. Rosenfeld G, Brown J, Vos PM, Leipsic J, Enns R, Bressler B. Prospective comparison of standard- versus low-radiation-dose CT enterography for the quantitative assessment of Crohn disease. AJR Am J Roentgenol. 2018;210(2):W54-W62. doi:10.2214/AJR.17. 18296

3. Chang R, Xu JX, Dong HP, et al. Value of CT spectral imaging in the evaluation of Crohn's disease activity. Diagnostic Theory Pract. 2019;4:432-435.

4. De Kock I, Delrue L, Lecluyse C, Hindryckx P, De vos M, Villeirs G. Feasibility study using iodine quantification on dual-energy CT enterography to distinguish normal small bowel from active inflammatory Crohn's disease. Acta Radiol. 2019;60(6):679-686. doi:10.1177/ 0284185118799508

5. Kim YS, Kim SH, Ryu HS, Han JK. Iodine quantification on spectral detector-based dual-energy CT enterography: correlation with Crohn's disease activity index and external validation. Korean J Radiol. 2018;19(6):1077-1088. doi:10.3348/kjr.2018.19.6.1077

6. Peng JC, Feng Q, Zhu J, et al. Usefulness of spectral computed tomography for evaluation of intestinal activity and severity in ileocolonic Crohn's disease. Therap Adv Gastroenterol. 2016;9(6): 795-805. doi:10.1177/1756283X16668309

7. Fulwadhva UP, Wortman JR, Sodickson AD. Use of dual-energy CT and iodine maps in evaluation of bowel disease. Radiographics. 2016;36(2):393-406. doi:10.1148/rg.2016150151

8. Lee SM, Kim SH, Ahn SJ, Kang HJ, Kang JH, Han JK. Virtual monoenergetic dual-layer, dual-energy CT enterography: optimization of keV settings and its added value for Crohn's disease. Eur Radiol. 2018;28(6):2525-2534. doi:10.1007/s00330-017-5215-z

9. Taguchi N, Oda S, Kobayashi T, et al. Advanced parametric imaging for evaluation of Crohn's disease using dual-energy computed tomography enterography. Radiol Case Rep. 2018;13(3):709-712. doi:10. 1016/j.radcr.2018.04.002

10. Wang CL, Han D, Huang YL. Feasibility study of dual energy CT spectral curve in evaluating the activity of Crohn's disease of colon. J Clin Radiol. 2019;8:1443-1448.

11. Liu CR. Feasibility of dual source CT dual energy technique in evaluating the activity of Crohn's disease. Kunming: Kunming Medical University; 2016: 18-21.

12. Javadi S, Elsherif S, Bhosale P, et al. Quantitative attenuation accuracy of virtual non-enhanced imaging compared to that of true non-enhanced imaging on dual-source dual-energy CT. Abdom Radiol. 2020;45(4):1100-1109. doi:10.1007/s00261-020-02415-8 across all disease areas. The manuscript management system is completely online and includes a very quick and fair peer-review system, which is all easy to use. Visit http://www.dovepress.com/ testimonials.php to read real quotes from published authors. 